\title{
Santhica 23 et 27 : deux variétés de chanvre (Cannabis sativa L.) sans $\Delta-9$-THC
}

\section{Santhica 23 and $27:$ two varieties of hemp (Cannabis sativa L.) without $\Delta-9-T H C$}

\section{Gilbert FOURNIER $^{(1) *}$, Olivier BEHEREC ${ }^{(2)}$, Sylvestre BERTUCELLI ${ }^{(2)}$}

(1) Laboratoire de Pharmacognosie, UMR 8076, CNRS (BioCIS), Faculté de Pharmacie, 5, rue J.-B. Clément - F-92290 Châtenay-Malabry (2) Fédération Nationale des Producteurs de Chanvre (FNPC), 20, rue Paul Ligneul - F-72000 Le Mans

*Auteur à qui adresser la correspondance : Professeur Gilbert FOURNIER, Laboratoire de Pharmacognosie, UMR 8076, CNRS (BioCIS), Faculté de Pharmacie, 5, rue J.-B. Clément - F-92296 Châtenay-Malabry Cedex Tél : 0146835597 - Fax : 0146835710 - E-mail : gilbert.fournier@cep.u-psud.fr

(Reçu le 3 mars 2004 ; accepté le $1^{\text {er juin 2004) }}$

\section{RÉSUMÉ}

Le cannabigérol $(C B G)$ est le précurseur biogénétique de tous les autres cannabinoüdes, substances spécifiques du chanvre. En 1987 a été identifié pour la première fois en France un nouveau chimiotype dont le CBG est le cannabinoïde majoritaire. Les teneurs de cette substance évoluent au cours du développement de la plante pour atteindre un maximum lors de la fin de la floraison. Au cours de la même période le $\Delta$-9-tétrahydrocannabinol ( $\Delta-9-T H C)$ n'a pas été retrouvé. La biogenèsé des cannabinoïdes semble donc s'être précocement arrêtée. Deux nouvelles variétés (Santhica 23 et 27) présentant ce profil cannabinoïdique ont été créées. Ne renfermant pas de $\Delta-9-T H C$, elles ne présentent aucune propriété psychotrope. Il est proposé de les considérer comme des «variétés à fibres de seconde génération».

\section{MOTS-CLÉS}

Chanvre, Cannabis sativa, cannabigérol, chimiotype, santhica.

\section{SUMMARY}

Cannabigerol $(C B G)$ is the biogenetic precursor of all the others cannabinoids, specific substances of hemp. In 1987, a new chemotype whose CBG is the dominant cannabinoide, was identified for the first time in France. The amount of this substance increases during the development of the plant to reach a maximum at the time of the end of flowering. During the same period the $\Delta-9$-tetrahydrocannabinol $(\triangle-9-T H C)$ was not found. The biogenesis of the cannabinoids seems to have stopped precociously. Two new varieties (Santhica 23 and 27) presenting this cannabinoidic profile were created. Not containing $\triangle-9-T H C$, they do not present any psychotropic properties. It is proposed to regard them as "second generation varieties of fibres".

\section{KEY-WORDS}

Hemp, Cannabis sativa, cannabigerol, chemotype, santhica. 


\section{Introduction}

Dans un article récemment publié, ont été rappelés les différents travaux relatifs à la biosynthèse des cannabinoïdes (1). La première étape consiste en une réaction de condensation du pyrophosphate de géranyle avec l'acide olivétolique pour former le cannabigérol (CBG) lui-même précurseur du cannabichromène (CBC), du cannabidiol (CBD) ou du $\Delta-9$-tétrahydrocannabinol ( $\Delta$ 9-THC) (2-11). A chaque étape, des enzymes catalysent la réaction (12-14).

Selon la proportion de divers cannabinoïdes, et donc selon l'activité enzymatique, il est désormais classique de distinguer plusieurs chimiotypes chez le chanvre. Ainsi, selon les proportions relatives de $\Delta-9-T H C$ et de $\mathrm{CBD}$, on distingue les chimiotypes «Drogue» ( $\Delta-9-$ THC $>$ CBD), «Drogue intermédiaire» (teneurs en $\Delta$ 9-THC et en CBD identiques) et «Fibres» ( $\Delta-9-\mathrm{THC}$ $<$ CBD) $(5,15-20)$.

Dans ces différents cas, la biosynthèse des cannabinoïdes va jusqu'à son terme. Il n'en est pas de même pour un chimiotype, décrit par l'un de nous, dans lequel la biosynthèse est quasiment arrêtée au stade du CBG (21). Les variétés «Santhica» 23 et 27 présentent cette caractéristique.

L'objet de ce travail est d'étudier l'évolution de la teneur en cannabinoïdes, notamment en $\mathrm{CBG}$, au cours de la croissance et selon la partie prélevée des plantes des variétés Santhica 23 et 27.

\section{Les variétés Santhica 23 et Santhica 27}

Santhica 23 et 27 sont deux variétés sélectionnées de chanvre obtenues par la Fédération Nationale des Producteurs de Chanvre (FNPC). Elles sont inscrites sur la liste des variétés pouvant bénéficier de paiements à la surface de la part de la Communauté Economique Européenne : Santhica 23 depuis l'année 1997 ; Santhica 27 depuis l'année 2002 [Règlements (CE) 2860/2000, 327/2002 et 335/2003] (22-24). Par ailleurs, selon le Code de la Santé Publique, la culture de la variété Santhica 23 est autorisée en France depuis 1997 (25), celle de la variété Santhica 27 depuis mars 2004 (26).

Du point de vue de leur composition chimique, ces variétés se caractérisent par la présence de CBG comme cannabinoïde dominant. La teneur en CBD ne dépasse pas $0,1 \%$. Ne renfermant pas de tétrahydrocannabinols (formes acides et neutres des $\Delta-9-\mathrm{THC}$ et $\Delta-8$-THC), elles ne présentent aucune propriété psychotrope.

\section{Méthodologie}

Les cultures de chanvre sont établies sur la base de $50 \mathrm{~kg}$ de semences à l'hectare, soit 250 à 300 plantes au $\mathrm{m}^{2}$. L'échantillonnage et les dosages des cannabinoïdes ont été effectués selon la procédure officielle (annexe $\mathrm{C}$ du règlement $(\mathrm{CE}) \mathrm{n}^{\circ} 1177 / 2000$ de la Commission du 31 mai 2000) (27).

Pour étudier l'évolution de la teneur en cannabinoïdes au cours du développement des plantes, les prélèvements sont effectués tous les 2 ou 3 jours ( 3 prélèvements par semaine) et concernent les tiers supérieurs de 200 plantes. Le traitement des échantillons prélevés et le dosage des cannabinoïdes $(\Delta-9-\mathrm{THC}$ et $\mathrm{CBG})$ par chromatographie en phase gazeuse (CPG) sont réalisés selon des protocoles décrits dans la méthode officielle européenne (27).

\section{Échantillonnage}

Les prélèvements, effectués pendant la journée, concernent les tiers supérieurs de 200 plantes. Le séchage est effectué à une température inférieure à $70^{\circ} \mathrm{C}$. Les échantillons secs sont débarrassés des tiges et des graines puis broyés (poudre demi-fine).

\section{Analyse du contenu en can- nabinoïdes}

- Réactifs : $\Delta-9$-THC et squalène (étalon interne) chromatographiquement purs ; solution d'extraction : solution à $35 \mathrm{mg}$ de squalène / 100 $\mathrm{ml}$ d'hexane.

- Extraction des cannabinoïdes : dans un tube, peser $100 \mathrm{mg}$ de la poudre préparée ci-dessus et extraire par $5 \mathrm{ml}$ de solution d'extraction. Placer dans un bac à ultrasons pendant $20 \mathrm{~min}$. Centrifuger. Prélever le surnageant et injecter $1 \mu l$.

- Appareillage : chromatographe en phase gazeuse muni d'un détecteur à ionisation de flamme et injecteur split/splitless. Colonne capillaire en verre de $25 \mathrm{~m}$ de long et $0,22 \mathrm{~mm}$ de diamètre. Phase apolaire de type $5 \%$ phényl-méthyl-siloxane. Température du four : $260^{\circ} \mathrm{C}$.

- Gamme d'étalonnage : quatre points : 0,04 - 0,10 0,25 et $0,50 \mathrm{mg} / \mathrm{ml}$ de $\Delta-9-\mathrm{THC}$ en solution d'extraction.

Les résultats sont exprimés en grammes de cannabinoïdes pour 100 grammes d'échantillon d'analyse, séché jusqu'à poids constant.

Le CBG n'étant pas commercialisé, il n'est pas possible d'en disposer pour réaliser une gamme d'étalonnage. Les teneurs en CBG sont donc ici déterminées en 
se référant à la gamme d'étalonnage du $\Delta-9-T H C$, cannabinoïde chimiquement et chromatographiquement proche.

\section{Résultats et discussion : évolution de la teneur en cannabinoïdes au cours de la croissance et selon la partie prélevée}

L'évolution des teneurs en cannabinoïdes au sein des deux variétés Santhica 23 et 27 est strictement parallèle. A titre d'exemple, seuls sont ici présentés les résultats obtenus pour la variété Santhica 27. A aucun moment il n'a été mis en évidence la présence de $\Delta$-9THC (ni de CBD) $\left(<10^{-5} \%\right)$ (Figure 1$)$.

L'évolution de la teneur en CBG depuis la levée des plantes est reproduite sur la Figure 1. La teneur en CBG varie au cours du cycle végétatif de la plante. Ainsi, la teneur en CBG croît assez régulièrement jusqu'à la fin de la floraison (75 à 80 jours après la levée). A partir de ce stade, la teneur en CBG est beaucoup plus fluctuante ; ceci est probablement lié à la perte plus ou moins importante d'une partie des feuilles, augmentant ainsi la proportion de l'inflorescence (classiquement, la teneur en cannabinoïdes est 2 à 4 fois plus élevée dans les inflorescences que dans les feuilles).

En analysant séparément les feuilles et les inflorescences, les variations de la teneur en CBG au cours du développement de la plante (Figure 2) sont comparables à celles déjà constatées pour le $\Delta-9-\mathrm{THC}$ et le CBD chez d'autres variétés (28). Lorsque l'on compare, sur la même période, les inflorescences et les feuilles : les teneurs en CBG y sont en moyenne trois fois plus élevées dans les premières que dans les secondes (variation d'un facteur 1,5 à 5,5 ).

\section{Conclusion}

Les industries utilisatrices de Chanvre disposent désormais de variétés sans tétrahydrocannabinols $(\Delta-9-\mathrm{THC}$ et $\Delta-8$-THC), il s'agit des variétés Santhica 23 et 27 . La biosynthèse des cannabinoïdes y est quasiment arrêtée au cannabigérol (CBG). L'intérêt de ces variétés est important en terme de santé publique puisqu'elles ne présentent aucune propriété psychoactive, celle-ci étant liée à la présence des tétrahydrocannabinols. Par ailleurs, les caractéristiques agronomiques et les critères de qualité de ces variétés (notamment leurs rendements en fibres corticales) sont comparables à ceux des autres variétés industrielles autorisées (29). Santhica 23 et 27, de par leurs utilisations et du fait de leur composition en cannabinoïdes, doivent désormais être considérées comme les premières «variétés à fibres de seconde génération».

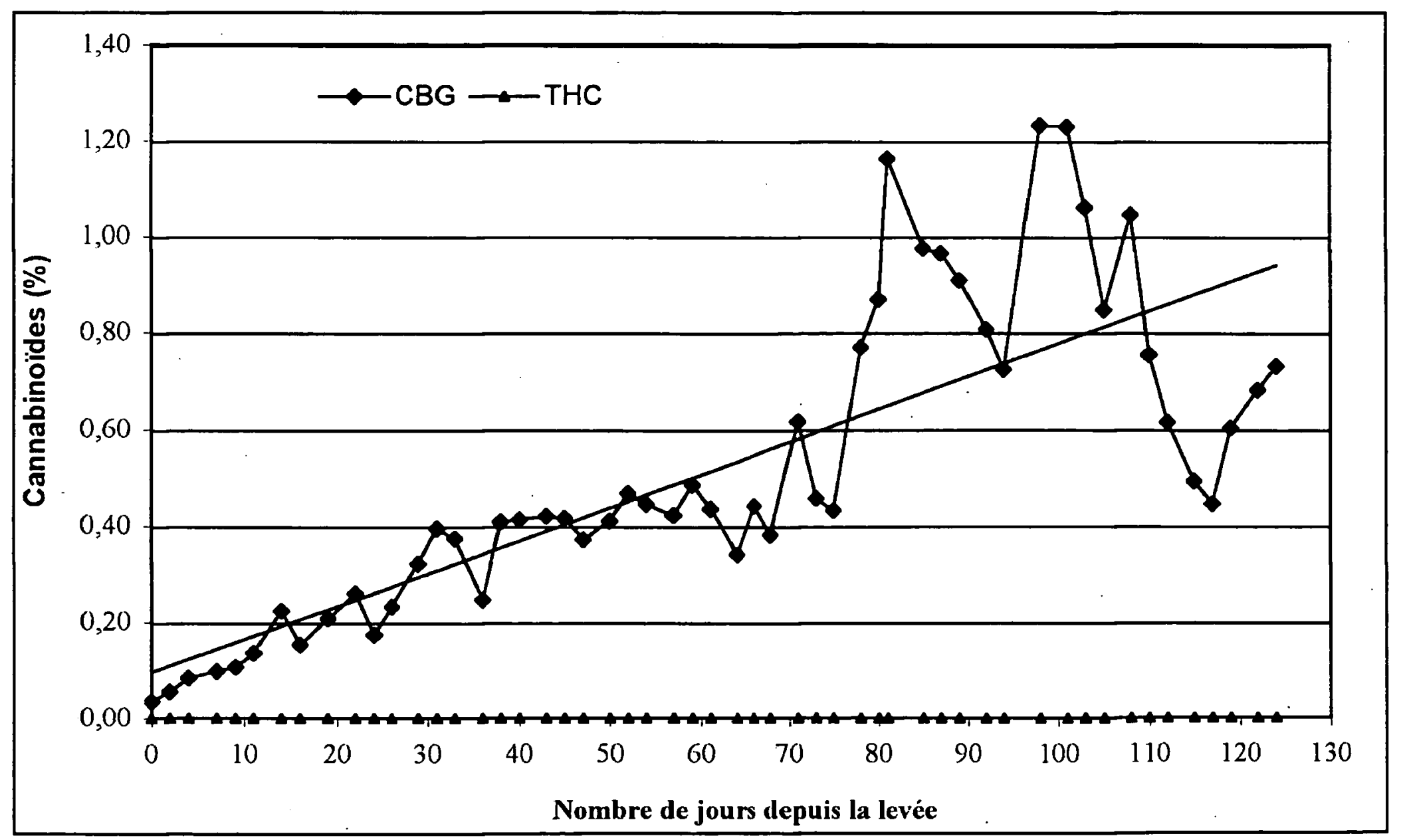

Figure 1 : Évolution des teneurs en $\triangle-9-T H C$ et en CBG au cours de la croissance. Variété Santhica 27 (feuilles et inflorescences). 


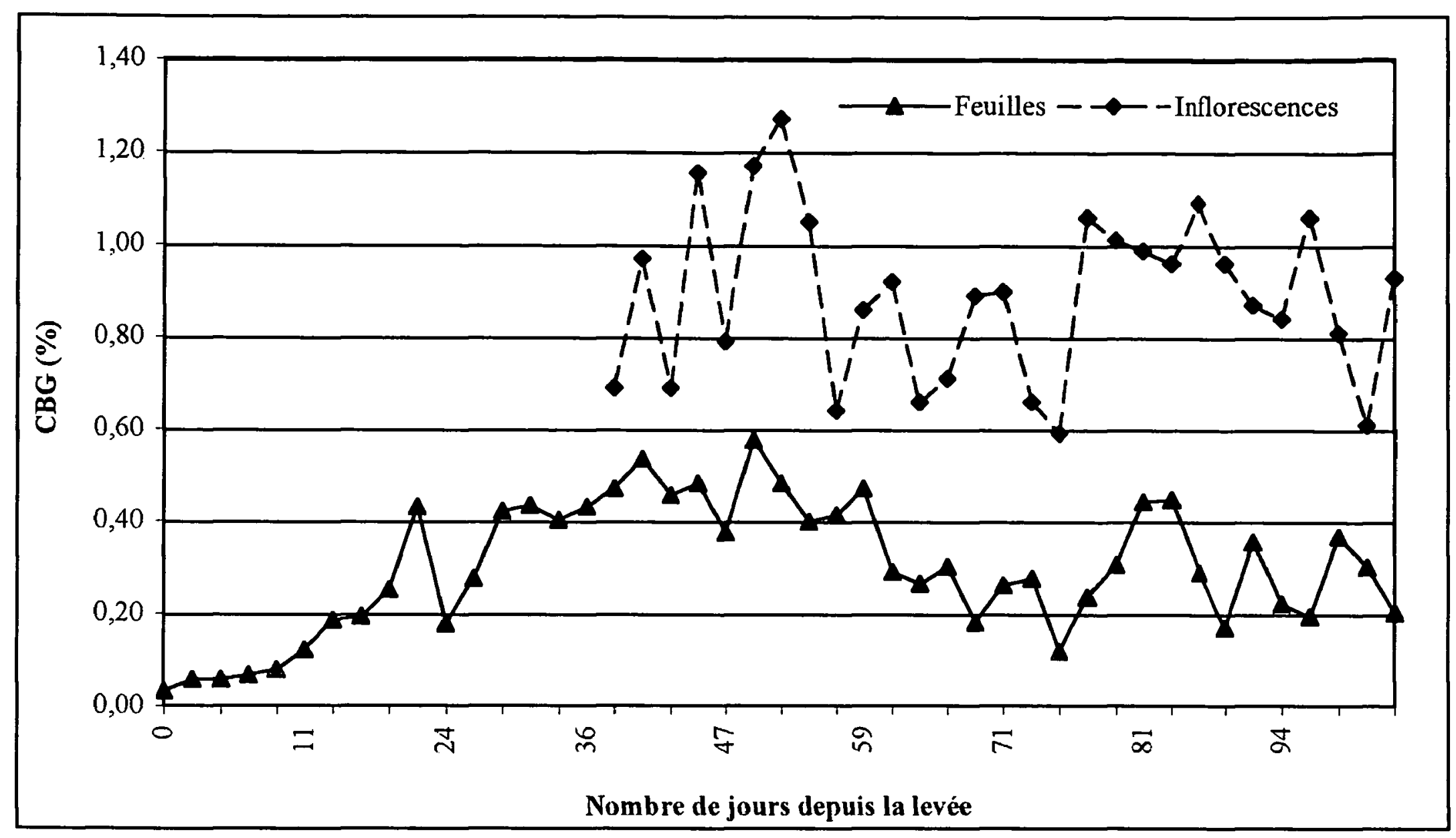

Figure 2 : Évolution des teneurs en CBG dans les feuilles et dans les inflorescences au cours de la croissance - Variété Santhica 27.

\section{Références}

1. de Meijer E.P.M., Magatta M., Carboni A., Crucitti P., Moliterni V.M.C., Ranalli P., Mandolino G. The inheritance of Chemical Phenotype in Cannabis sativa $\mathrm{L}$. Genetics $2003 ; 163: 335-46$.

2. Gaoni Y., Mechoulam R. Cannabichromene, a new active principle in hashish. Chem. Commun. 1966; $1: 20-1$.

3. Mechoulam R. Marihuana Chemistry. Science 1970 ; 68(3936) : 1159-68.

4. Shoyama Y., Yagi M., Nishioka I., Yamauchi T. Biosynthesis of cannabinoid acids. Phytochemistry $1975 ; 14: 2189-92$.

5. Fournier G., Paris M. Détermination de chimiotypes à partir des cannabinoïdes chez le chanvre à fibres monoïque (Cannabis sativa L.). Possibilités de sélection. Physiol. Vég. 1980 ; 18(2) : 349-56.

6. Kajima M., Piraux M. The biogenesis of cannabinoids in Cannabis sativa. Phytochemistry $1982 ; 21(1)$ : 67-9.

7. Shoyama Y., Hirano H., Nishioka I. Biosynthesis of propyl cannabinoid acid and its biosynthetic relationship with pentyl and methyl cannabinoïde acids. Phytochemistry 1984 ; 23(9) : 1909-12.

8. Hanus L. Biogenesis of cannabinoid substances in the plant. Acta Univ. Olomuc., Fac. Med. 1987 ; 116 : 47-53.

9. Vogelmann A.F., Turner J.C., Mahlberg P.G. Cannabinoid occurrence in seedlings of Cannabis sativa $\mathrm{L}$ : quantitation in seedlings of known age and primary leaf length. Bot. Gaz. 1987 ; 148(3) ; 468-74.

10. Morimoto S., Taura F., Shoyama Y. Biosynthesis of cannabinoids in Cannabis sativa L. Current Topics in Phytochemistry $1999 ; 2$ : 103-13.
11.Fellermeier M., Eisenreich W., Bacher A., Zenk M.H. Biosynthesis of cannabinoids. Incorporation experiments with 13C-labelled glucose. Eur. J. Biochem. $2001 ; 268$ : 1596-604.

12. Taura F., Morimoto S., Shoyama Y. Purification and characterization of cannabidiolic-acid synthase from Cannabis sativa L. J. Biol. Chem. 1996 ; 271 : 17411-6.

13. Morimoto S., Komatsu K., Taura F., Shomaya Y. Purification and characterization of cannabichromenic acid synthase from Cannabis sativa. Phytochemistry $1998 ; 49(6)$ : 1525-9.

14. Fellermeier M., Zenk M.H. Prenylation of olivetolate by a hemp transferase yields cannabigerolic acid, the precursor of tetrahydrocannabinol. Fed. Eur. Biochem. Soc. 1998 ; 427 : 283-5.

15. Fetterman P.S., Keith E.S., Waller C.W., Guerrero O., Doorenbos N.J., Quimby M.W. Mississippi-Grown Cannabis sativa L. : preliminary observation on chemical definition of phenotype and variations in tetrahydrocannabinol content versus age, sex, and plant part. J. Pharm. Sci. $1971 ; 60$ : 1246-9.

16. Small E. The species problem in Cannabis: Science \& Semantics. Corpus ed. Toronto 1979 ; I-218p, II-156p.

17. Turner C.E., Elsohly M.A., Cheng P.C., Lewis G. Constituents of Cannabis sativa $\mathrm{L}$., XIV : Intrinsic problems in classifying cannabis based on a single cannabinoid analysis. J. Nat. Prod. 1979 ; 42 : 317-9.

18. Fournier G. Les chimiotypes du chanvre (Cannabis sativa $\mathrm{L}$.). Intérêt pour un programme de sélection. Agronomie $1981 ; 1(8)$ : 679-88. 
19. Brenneisen R., Elsohly M.A. Chromatographic and spectroscopic profiles of Cannabis of different origins : Part I. J. Forensic Sc. 1988 ; 33(6) : 1385-404.

20. Fournier G. La sélection du Chanvre à fibres (Cannabis sativa L.) en France. Chanvre et THC. C. R. Acad. Agric. Fr. $2000 ; 86(7)$ : 209-17.

21. Fournier G., Richez-Dumanois C., Duvezin J., Mathieu J.P., Paris M. Identification of a new chemotype in Cannabis sativa : cannabigerol-dominant plants, biogenetic and agronomic prospects. Planta Med. 1987 ; 53(3) : 277-80.

22. Journal officiel des Communautés européennes Règlement (CE) $n^{\circ}$ 2860/2000 de la Commission du 27 décembre 2000 modifiant le règlement (CE) $n^{\circ}$ $2316 / 1999$ portant modalités d'application du règlement (CE) $\mathrm{n}^{\circ} 1251 / 1999 \mathrm{du}$ Conseil instituant un régime de soutien aux producteurs de certaines cultures arables pour y inclure le lin et le chanvre destinés à la production de fibres, préciser les règles concernant les superficies gelées et modifier les superficies de base pour la Grèce et le Portugal.

23. Journal officiel des Communautés européennes Règlement (CE) $n^{\circ} 327 / 2002$ de la Commission du 21 février 2002 modifiant le règlement (CEE) n ${ }^{\circ} 2316 / 1999$ portant modalités d'application du règlement (CE) $n^{\circ}$ $1251 / 1999$ du Conseil instituant un régime de soutien aux producteurs de certaines cultures arables.
24. Journal officiel des Communautés européennes Règlement (CE) $n^{\circ} 335 / 2003$ de la Commission du 21 février 2003 modifiant le règlement (CEE) $n^{\circ} 2316 / 1999$ portant modalités d'application du règlement (CE) $n^{\circ}$ 1251/1999 du Conseil instituant un régime de soutien aux producteurs de certaines cultures arables.

25. Code de la Santé Publique - Arrêté du 27 mai 1997 modifiant l'arrêté du 22 août 1990 portant application de l'article R. 5181 du code de la santé publique pour le cannabis - J.O. du 31 mai 1997.

26. Journal officiel de la République française - Arrêté du 24 février 2004 modifiant l'arrêté du 22 août 1990 portant application de l'article R.5181 du code de la santé publique pour le cannabis - J.O. du 21 mars 2004.

27. Journal officiel des Communautés européennes Règlement (CE) $n^{\circ} 1177 / 2000$ de la Commission du 31 mai 2000 modifiant le règlement (CEE) $n^{\circ} 1164 / 89$ relatif aux modalités d'aide concernant le lin textile et le chanvre.

28. Fournier G. Intérêt du rapport $\triangle-9-T H C$ / CBD dans le contrôle des cultures de chanvre industriel. Ann. Toxicol. Anal. 2003 ; 15(4) : 250-9.

29. Fournier G. A propos de la teneur en $\Delta-9-$ THC dans les variétés de chanvre à fibres cultivées en France. Ann. Toxicol. Anal. $2003 ; 15(1): 30-4$. 